\title{
Experimental Strategy for Characterization of Essential Dynamical Variables in Oscillatory Systems: Effect of Double-Layer Capacitance on the Stability of Electrochemical Oscillators
}

\author{
István Z. Kiss, ${ }^{\dagger}$ Zoltán Kazsu,, and Vilmos Gáspár*, \\ Department of Chemical Engineering, University of Virginia, 102 Engineers' Way, Charlottesville, Virginia \\ 22904, and Institute of Physical Chemistry, University of Debrecen, 4010 Debrecen, PO Box 7, Hungary
}

Received: July 4, 2005; In Final Form: August 26, 2005

\begin{abstract}
An experimentally accessible algorithm for changing the time scale associated with a dynamical variable is proposed. In general, a differential controller can be applied to (a) identify the essential species in oscillatory systems and (b) explore their role in the feedback loops. Here, we report on classifying electrochemical oscillators by changing the time scale over which the electrode potential varies; the type of different electrochemical oscillators is identified based on whether the controlled modification of pseudo-capacitance induces or suppresses current oscillations.
\end{abstract}

\section{Introduction}

A general (although not exclusive) feature of oscillatory chemical systems is the existence of essential species that form destabilizing (usually fast autocatalytic or self-inhibitory) and stabilizing (slow inhibitory) feedback loops. ${ }^{1-5}$ These loops can be identified with stoichiometric network analysis ${ }^{2}$ of the underlying complex reaction mechanism. Such analysis has proven to be a valuable procedure in the classification of the myriad of oscillatory chemical reactions ${ }^{6}$ and electrochemical systems $^{7}$ as well.

In oscillatory electrochemical systems the dynamical behavior depends not only on the surface concentration of electrochemically active species but also on the potential drop across the double-layer. ${ }^{8-10}$ When the electrode potential plays an essential role in the origin of current oscillations, the electrochemical system exhibits negative differential resistance (NDR) in a certain region of the overpotential. The electrode potential can play the role of either a fast activator (N-NDR) or a slow inhibitor (S-NDR) species. ${ }^{7,9}$ The "N" and "S" letters refer to the characteristic shapes through which the negative slope of a polarization (current vs potential) curve may develop. Furthermore, in some systems, the N-NDR character is hidden (HNNDR). These systems can oscillate even under galvanostatic conditions (N-NDR systems show bistability only). ${ }^{7}$

In recent years, an operational procedure-based on studying the dependence of dynamics on the cell resistance-was proposed to identify the type of an electrochemical oscillator. ${ }^{7}$ However, the effect of changing the resistance of a cell is not always straightforward. Chemically, it can be achieved by changing the composition of the electrolyte that, however, might affect some kinetic parameters. Electronically, the cell resistance can be modified by adding series resistance. In addition, the solution resistance may be compensated by an electronic feedback loop (current drop compensation). Such changes, however, might introduce some global coupling to the system., ${ }^{9,11}$

In this paper, we propose an experimentally accessible method-based on a "differential controller"-for fine-tuning the

* Corresponding author. E-mail: gasparv@ delfin.unideb.hu.

University of Virginia.

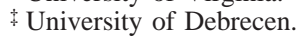

time scale over which the concentration of chemical species or the value of other dynamical variables can vary. Since oscillations require the accurate timing of the feedback loops, the periodic behavior can be suppressed or induced by changing the time scales. We demonstrate-using electrochemical systems as testbeds-how the essential species can be identified and how their role can be explored with the application of the controller. In an unperturbed electrochemical system, the time scale of the variation of the electrode potential is set by the double-layer capacitance of the electrode. Therefore, a "differential controller" has been designed to change the capacitance accordingly. The effect of such changes is investigated in three prototype electrochemical systems: (i) the electrodissolution of $\mathrm{Cu}$ in phosphoric acid, ${ }^{12}$ (ii) the electrodissolution of $\mathrm{Ni}$ in sulfuric acid, ${ }^{13}$ and (iii) the electrodepositon of $\mathrm{Zn}$ in acidic electrolyte. ${ }^{14-16}$ In all these systems, numerical simulations and experiments are carried out, and the role of electrode potential in the oscillatory mechanism is clarified.

\section{Methods, Models, and Experiments}

Differential Controller: A Versatile Tool for Changing Time Scales. The differential controller is widely used for process control in engineering. ${ }^{17}$ The main features of the method can be summarized as follows. Let us take an $n$-variable system with variables $x_{n}$. In most cases, the dynamical behavior of the system can be described by a set of ordinary differential equations:

$$
\epsilon_{k} \mathrm{~d} x_{k} / \mathrm{d} t=f_{k}\left(x_{1}, x_{2}, \ldots, x_{n}\right)
$$

where $f_{k}$ are component functions that depend on variables $k=$ $1,2, \ldots, n$, and $\epsilon_{k}$ defines the time scale for the variation of the $k$ th variable. (We note that time scales $\epsilon_{k}$ strongly depend on the specific forms of functions $f_{k}$.) A differential controller with feedback strengths $\alpha_{k}$ can often be introduced in such a way that the equations become

$$
\epsilon_{k} \mathrm{~d} x_{k} / \mathrm{d} t=f_{k}\left(x_{1}, x_{2}, \ldots, x_{n}\right)+\alpha_{k} \mathrm{~d} x_{k} / \mathrm{d} t
$$


which can be rearranged as follows

$$
\left(\epsilon_{k}-\alpha_{k}\right) \mathrm{d} x_{k} / \mathrm{d} t=f_{k}\left(x_{1}, x_{2}, \ldots, x_{n}\right)
$$

The characteristic difference between eqs 3 and 1 appears on the left-hand side of the equations. While $\epsilon_{k}$ values are fixed, the new time scale values $\epsilon_{k}^{\prime}=\left(\epsilon_{k}-\alpha_{k}\right)$ can be fine-tuned by varying the control gains. (Note, however, that the controller does not modify the stationary solution of eq 1 , but it might change the stability of the steady states. ${ }^{18}$ ) The differential controller (eq 2) implemented only for a single variable can be applied to change the time scale corresponding to that variable without changing any other parameters in the system.

Design of Differential Controller for Electrochemical Systems. In electrochemical systems the specific double-layer capacitance $C_{\mathrm{d}}\left(\mathrm{F} / \mathrm{cm}^{2}\right)$ sets the inherent time scale for the variation of the potential drop $e$ through the double-layer as shown by the charge balance equation ${ }^{9}$ for potentiostatic mode of operation:

$$
C_{\mathrm{d}} \frac{\mathrm{d} e}{\mathrm{~d} t}=-j_{\mathrm{F}}(c, \theta, e, \ldots)+\frac{V-e}{A R_{\Omega}}
$$

where $j_{\mathrm{F}}$ is the Faradaic current density, $c$ is the concentration of some electroactive species, $\theta$ is surface coverage, $V$ is the applied circuit potential, $A$ is the surface area of the electrode, and $R_{\Omega}$ is the cell resistance. In typical potentiostatic experiments, $V$ is controlled externally and the current $i=(V-e) /$ $R_{\Omega}$ is measured. For the implementation of the differential controller for variable $e$ in the form of eq 2, we take $V$ as the control parameter and $i$ as the control variable, and invoke a recursive version of the control algorithm. In experiments, the circuit potential can be varied around $V_{\mathrm{o}}$ according to the following equation:

$$
\begin{array}{r}
V=V_{\mathrm{o}}+\gamma \frac{\mathrm{d} V}{\mathrm{~d} t}-\gamma R_{\Omega} \frac{\mathrm{d} i}{\mathrm{~d} t}=V_{\mathrm{o}}+\gamma \frac{\mathrm{d} V}{\mathrm{~d} t}-\gamma R_{\Omega} \frac{\mathrm{d}}{\mathrm{d} t}\left(\frac{V-e}{R_{\Omega}}\right)= \\
V_{\mathrm{o}}+\gamma \frac{\mathrm{d} e}{\mathrm{~d} t}
\end{array}
$$

where $\gamma$ is the control gain. Combining eqs 5 and 4 results in the following dynamical equation for the circuit potential of the cell under recursive control:

$$
\left(C_{\mathrm{d}}-\frac{\gamma}{A R_{\Omega}}\right) \frac{\mathrm{d} e}{\mathrm{~d} t}=-j_{\mathrm{F}}(c, \theta, e \ldots)+\frac{V_{\mathrm{o}}-e}{A R_{\Omega}}
$$

The term $C_{\mathrm{d}}{ }^{\prime}=-\gamma /\left(\mathrm{A} R_{\Omega}\right)$ on the left-hand side of eq 6 acts as a specific pseudo-capacitance. The term $\left(C_{\mathrm{d}}+\mathrm{C}_{\mathrm{d}}{ }^{\prime}\right)$ in eq 6 plays the same role as the new time scale $\epsilon_{k}^{\prime}=\left(\epsilon_{k}-\alpha_{k}\right)$ in eq 3. In the following sections, we shall study the effect of the specific pseudo-capacitance $C_{\mathrm{d}}{ }^{\prime}$ on the stability of three prototype electrochemical oscillators. In the Experimental Section $C_{\mathrm{d}} *=$ $C_{\mathrm{d}}{ }^{\prime} A(\mathrm{~F})$ is used to denote pseudo-capacitance.

Model of Copper Electrodissolution. The Koper-Gaspard dimensionless model ${ }^{19}$ was used to simulate the main dynamical features of $\mathrm{Cu}$ dissolution in phosphoric acid solution:

$$
\begin{gathered}
C_{\mathrm{d}} \frac{\mathrm{d} e}{\mathrm{~d} t}=\frac{V-e}{R}-120 k(e) u \\
\frac{\mathrm{d} u}{\mathrm{~d} t}=-1.25 d^{1 / 2} k(e) u+2 d(w-u) \\
\frac{\mathrm{d} w}{\mathrm{~d} t}=1.6 d(2-3 w+u)
\end{gathered}
$$

where $V$ is the applied circuit potential, $e$ is the double-layer potential, $R$ is the series resistance, $d$ is the rotation rate, $u$ and $w$ are the dimensionless concentrations of some electroactive species, respectively, in the so-called surface and diffusion layers, and $k(e)$ is the potential dependent rate constant defined as

$$
k(e)=2.5 \theta^{2}+0.01 \exp [0.5(e-30)]
$$

where $\theta$ is related to the potential dependent fractional surface coverage by the electroactive species:

$$
\theta=\left\{\begin{array}{lr}
1 & \text { for } e \leq 35 \\
\exp \left[-0.5(e-35)^{2}\right] & \text { for } e>35
\end{array}\right\}
$$

The experimentally measured quantity, the current, is obtained as $i=(V-e) / R$. Dynamics of model eqs 7-9 have been studied in detail by Koper and Gaspard. ${ }^{19}$ For an appropriate range of parameters the model simulates well the dynamical behavior of $\mathrm{Cu}$ dissolution in phosphoric acid observed in our potentiostatic experiments. ${ }^{20,21}$ In this study we set $R=0.02$ and $d=$ 0.11913 , and the dynamical features are explored by systematically varying the circuit potential $V$ and the specific doublelayer capacitance $C_{\mathrm{d}}$.

Model of Nickel Electrodissolution. A dynamical model for the anodic electrodissolution of a nickel electrode is based on two dimensionless variables, the double-layer potential $e$ and the total surface coverage $\theta$ by $\mathrm{NiO}$ and $\mathrm{NiOH}$. The differential equations proposed by Haim et al. ${ }^{22}$ are as follows:

$$
\begin{gathered}
C_{d} \frac{\mathrm{d} e}{\mathrm{~d} t}=\frac{V-e}{R}-j_{\mathrm{F}}(\theta, e) \\
\Gamma \frac{\mathrm{d} \theta}{\mathrm{d} t}=\frac{\exp (0.5 e)}{1+C_{h} \exp (e)}(1-\theta)-\frac{b C_{h} \exp (2 e)}{c C_{h}+\exp (e)} \theta
\end{gathered}
$$

where $V$ is the dimensionless circuit potential, $R$ is the dimensionless series resistance, $\Gamma$ is the maximal surface coverage, and $j_{\mathrm{F}}$ is the Faradaic current density:

$$
j_{\mathrm{F}}=\left(\frac{C_{h} \exp (0.5 e)}{1+C_{h} \exp (e)}+a \exp (e)\right)(1-\theta)
$$

Equation 10a accounts for the charge balance in the doublelayer, while eq $10 \mathrm{~b}$ follows from the mass balance and rate equations for the electroactive species. The current is calculated as $i=(V-e) / R$. The parameter values $\left(C_{h}=1600, a=0.3\right.$, $b=6 \times 10^{-5}, c=1 \times 10^{-3}, \Gamma=0.01$, and $\left.R=50\right)$ were optimized to obtain dynamical features similar to those observed in experiments. ${ }^{11}$

Model of Zinc Electrodepostion. The Lee-Jorné model ${ }^{23}$ has been applied to simulate the dynamics of $\mathrm{Zn}$ electrodeposition under potentiostatic operation. The originally two-variable model that quantitatively describes the experimentally observed Sshaped polarization curve has been modified to incorporate the potential (IR) drop through the electrolyte and an external resistance in series with the electrochemical cell. The differential equations are as follows: 


$$
\begin{gathered}
C_{\mathrm{d}} \frac{\mathrm{d} e}{\mathrm{~d} t}=\frac{V-e}{R^{\prime}}-j_{\mathrm{F}}\left(e, \theta_{1}, \theta_{2}\right) \\
\Gamma_{1} \frac{\mathrm{d} \theta_{1}}{\mathrm{~d} t}=A_{1}\left(1-\theta_{1}-\theta_{2}\right)-A_{2} \theta_{1}-A_{4} \theta_{1} \theta_{2}-A_{6} \theta_{1} \\
\Gamma_{2} \frac{\mathrm{d} \theta_{2}}{\mathrm{~d} t}=A_{3} \theta_{2}\left(1-\theta_{1}-\theta_{2}\right)-A_{3}{ }^{\prime} \theta_{2}^{2}-A_{4} \theta_{1} \theta_{2}-A_{5} \theta_{2}+ \\
A_{6} \theta_{1} \quad(12 \mathrm{~b})
\end{gathered}
$$

where $e$ and $V$ are, respectively, the double-layer and circuit potentials, $R^{\prime}$ is the resistivity of the electrode $\left(\Omega \mathrm{cm}^{2}\right), \theta_{1}$ and $\theta_{2}$ are, respectively, the time-dependent fractional surface coverage by the adsorbed $\mathrm{H}$ and $\mathrm{Zn}^{+}$(the maximal values are $\Gamma_{1}$ and $\Gamma_{2}$ ), and $A_{k}$ are kinetic parameters. The Faradaic current density $j_{\mathrm{F}}$ is defined according to

$$
\begin{aligned}
j_{\mathrm{F}}\left(e, \theta_{1}, \theta_{2}\right) / F=- & A_{1}\left(1-\theta_{1}-\theta_{2}\right)-A_{2} \theta_{1}- \\
& A_{3} \theta_{2}\left(1-\theta_{1}-\theta_{2}\right)+A_{3}^{\prime} \theta_{2}^{2}-A_{5} \theta_{2}
\end{aligned}
$$

where $F$ is the Faradaic constant. The kinetic parameters were optimized by Lee and Jornéér and are as follows (in mol cm${ }^{-2}$ $\left.\mathrm{s}^{-1}\right): A_{1}=5.53 \times 10^{-7} \exp (-19.3 e), A_{2}=3 \times 10^{-9} \exp (-$ $29.3 e), A_{3}=2.45 \times 10^{-5} \exp (-33.8 e), A_{3}{ }^{\prime}=7.5 \times 10^{-5}$ $\exp (4.8 e), A_{4}=1 \times 10^{-6}, A_{5}=5.4 \times 10^{-8} \exp (-38.6 e)$, and $A_{6}=1 \times 10^{-9}$. The applied values of $\Gamma_{1}$ and $\Gamma_{2}$ were approximated by Epelboin et al..$^{14,15}$ from impedance measurements: $\Gamma_{1}=1.36 \times 10^{-7} \mathrm{~mol} \mathrm{~cm}^{-2}, \Gamma_{2}=9.067 \times 10^{-11} \mathrm{~mol}$ $\mathrm{cm}^{-2}$. To simulate the experimental observations, the current density is calculated as $j=(V-e) / R^{\prime}$. In this study, we set $R^{\prime}$ $=2.0 \Omega \mathrm{cm}^{2}$. The dynamical features are explored by systematically varying the circuit potential $V$ and the specific double-layer capacitance $C_{\mathrm{d}}$.

Numerical Tools. The ordinary differential equations (eqs 7,10 , and 12) were solved numerically with XPPAUT program package ${ }^{24}$ applying a fourth-order Runge-Kutta method with variable step size. Bifurcation diagrams were constructed with the built-in AUTO interface.

Experiments. The experiments on $\mathrm{Cu}$ and $\mathrm{Ni}$ electrodissolution and $\mathrm{Zn}$ electrodeposion were carried out in a standard three-electrode electrochemical cell equipped with a rotating disk working electrode (Radiometer EDI-101), calomel reference electrode, and Pt counter electrode. ${ }^{20}$ The working electrode was a $5 \mathrm{~mm}$ diameter $\mathrm{Cu}$ disk (rotated at $1500 \mathrm{rpm}$ ) in $85 \%$ phosphoric acid at $-5^{\circ} \mathrm{C}$, a $1 \mathrm{~mm}$ diameter stagnant Ni disk in $4 \mathrm{~mol} \mathrm{dm}{ }^{-3}$ sulfuric acid at $10{ }^{\circ} \mathrm{C}$, and a $7 \mathrm{~mm}$ diameter $\mathrm{Zn}$ electrode (rotated at $1000 \mathrm{rpm}$ ) in $0.72 \mathrm{~mol} \mathrm{dm}^{-3} \mathrm{ZnCl}_{2}+2.67$ mol dm ${ }^{-3} \mathrm{NH}_{4} \mathrm{Cl}$ buffer $(\mathrm{pH}=5,2)$ at $26{ }^{\circ} \mathrm{C}$ in the $\mathrm{Cu}, \mathrm{Ni}$, and $\mathrm{Zn}$ experiments, respectively.

The circuit potential was set and the current of the electrode was measured (sampling frequency: $200 \mathrm{~Hz}$ ) with a potentiostat (Electroflex EF-451). The working electrode was connected to the potentiostat through an external resistance $R_{\text {ext }}$; the total resistance $R_{\Omega}$ of the circuit is the sum of the external resistance and the cell resistance measured with impedance spectroscopy. ${ }^{21}$ The differential controller was implemented by using a Pascal program with real-time accuracy of $200 \mathrm{~Hz}$. The circuit potential was perturbed according to eq 5 using feedback gain $\gamma$, the time derivative of circuit potential and current, and the previously determined $R_{\Omega}$. The added pseudo-capacitance is calculated as $C_{\mathrm{d}}{ }^{*}=-\gamma / R_{\Omega}(\mathrm{F})$. Further experimental details are given in earlier publications. ${ }^{20,21}$
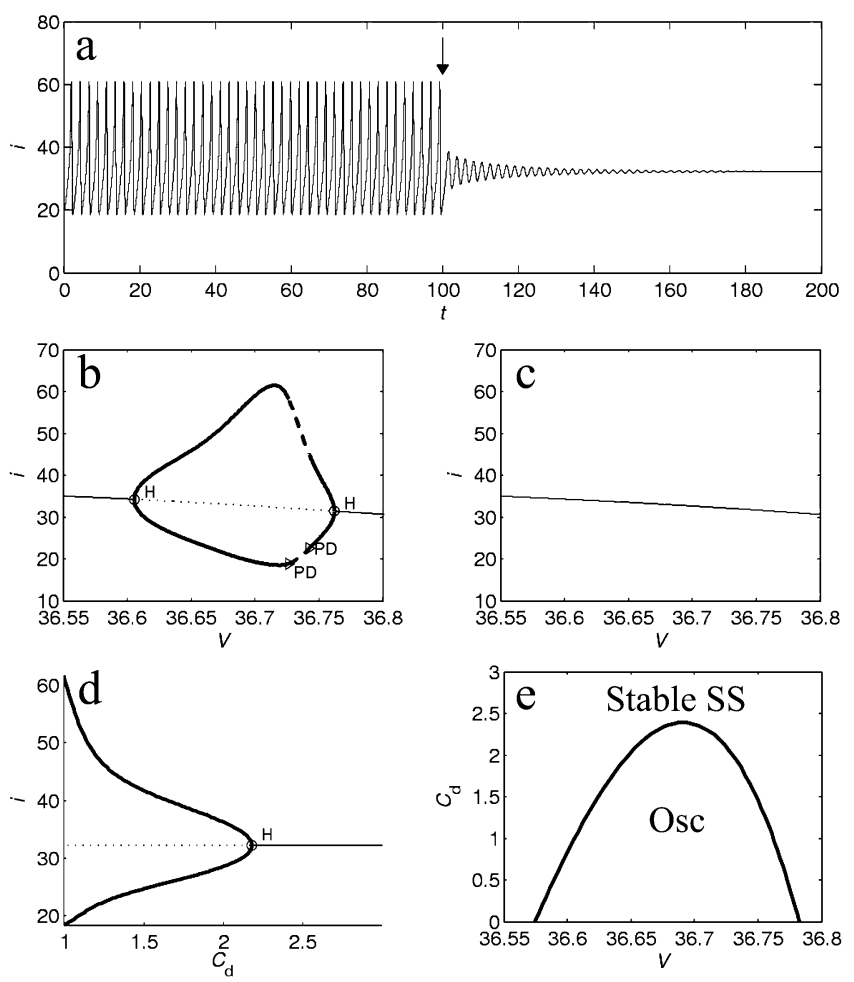

Figure 1. Simulations: Effect of changing the time scale associated with the electrode potential on current oscillations in $\mathrm{Cu}$ electrodissolution (N-NDR system). (a) Suppressing oscillations at $V=36.72$. At $t=100$ (shown by an arrow) the value of $C_{\mathrm{d}}$ is changed from 1 to 2.5 . (b) One parameter bifurcation diagram as a function of $V$ at $C_{\mathrm{d}}=1$ : solid thin line, stable steady state; dotted line, unstable steady-state; solid thick line, maximum and minimum current values of stable oscillatory solution; dashed thick line, maximum and minimum current values of unstable oscillatory solution; $\mathrm{H}$, Hopf-bifurcation; $\mathrm{PD}$, period doubling bifurcation. (c) Bifurcation diagram as a function of $V$ at $C_{\mathrm{d}}$ $=2.5$. (d) One parameter bifurcation diagram as a function of $C_{\mathrm{d}}$ at $V$ $=36.72$. (e) Two parameter bifurcation diagram showing the locus of Hopf bifurcations.

\section{Results}

3.1. The Effect of Changing the Pseudo-Capacitance on an N-NDR Oscillator: Copper Electrodissolution in Phosphoric Acid. For the electrodissolution of copper in phosphoric acid, the electrode potential was proposed to play the role of a fast activator species. ${ }^{19}$ Numerical simulations and experiments were carried out to confirm that the electrode potential is, indeed, an essential dynamical variable of this N-NDR type oscillator, and the effect of changing its time scale parameter was studied systematically.

Numerical Simulations. Figure 1a shows a typical oscillatory solution of the dimensionless model of $\mathrm{Cu}$ dissolution (eqs 7ac) at $V=36.72$. The current oscillations are suppressed by increasing the (dimensionless) double-layer capacitance from 1 to 2.5 at $t=100$. The oscillations die out with decreasing amplitude after a short transient period. The corresponding bifurcation diagrams as a function of circuit potential $V$ are shown in parts $\mathrm{b}$ and $\mathrm{c}$ of Figure 1 at $C_{\mathrm{d}}=1$ and 2.5, respectively. At $C_{\mathrm{d}}=1$, the steady state loses stability through a supercritical Hopf bifurcation. In a small region of the circuit potential, complex oscillations develop through period doubling bifurcations that eventually lead to chaos (not shown here). However, with an increase of $C_{\mathrm{d}}$ to 2.5 , both the periodic and complex oscillations disappear, and only stable steady-state solutions can be observed. It is seen from Figure 1d that the oscillations are destroyed through a supercritical Hopf bifurca- 

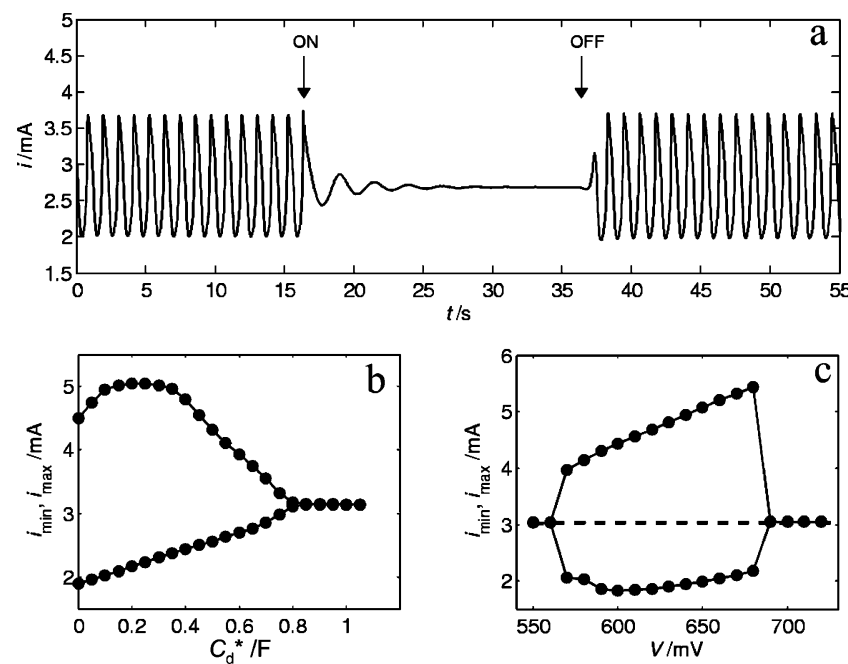

Figure 2. Experiments: Effect of changing the time scale associated with the electrode potential on current oscillations in $\mathrm{Cu}$ electrodissolution (N-NDR system). (a) Suppressing oscillations at $V_{\mathrm{o}}=590 \mathrm{mV}$ and $R_{\Omega}=85 \Omega$ by applying a pseudo-capacitance. Its value $\left(C_{\mathrm{d}}{ }^{*}=\right.$ $0.5 \mathrm{~F}$ ) is set with turning on the control algorithm for the period defined by the arrows. (b) One parameter bifurcation diagram at $V_{\mathrm{o}}=600 \mathrm{mV}$ and $R_{\Omega}=85 \Omega$ showing the minima and maxima of current oscillations as a function of the applied pseudo-capacitance $C_{\mathrm{d}}{ }^{*}$. (c) One parameter bifurcation diagrams as a function of the circuit potential $V_{\mathrm{o}}$ : solid line, $C_{\mathrm{d}}{ }^{*}=0 \mathrm{~F}$; dashed line, $C_{\mathrm{d}}{ }^{*}=2.0 \mathrm{~F}$.

tion at $C_{\mathrm{d}} \approx 2.2$. The locus of Hopf bifurcation points shown in Figure 1e divides the $C_{\mathrm{d}}$ vs $V$ parameter space into regions of oscillatory and stable stationary solutions. It is seen in this nonequilibrium phase diagram that there exists a maximal value of $C_{\mathrm{d}}$ (approximately 2.4) above which oscillations are suppressed at any circuit potential.

Experiments. Figure 2a shows that generating a large enough pseudo-capacitance $\left(C_{\mathrm{d}}{ }^{*}=0.5 \mathrm{~F}\right)$ effectively destroys the current oscillations during $\mathrm{Cu}$ electrodissolution. Note that although oscillations seemingly cease, yet a small control signal (not shown in the figure) is still required for stabilizing the steady state; after turning off the control algorithm (and thus resetting the double-layer capacitance), the current oscillations reappear with exactly the same amplitude and frequency as those before the control session. The maxima and minima of the experimentally observed current oscillations are shown in Figure $2 \mathrm{~b}$ as a function of the applied pseudo-capacitance. Oscillations are suppressed through decreasing amplitude around the bifurcation point at about $C_{\mathrm{d}} *=0.8 \mathrm{~F}$; the vanishing amplitude and the finite frequency (not shown in the figure) are indicative of a supercritical Hopf bifurcation similar to that found in simulations. The current oscillations could be suppressed in a large region of both experimental parameters $V$ and $C_{\mathrm{d}} *$. For example, Figure $2 \mathrm{c}$ shows the maxima and minima of current oscillations as a function of the circuit potential; oscillations appear at about $V=570 \mathrm{mV}$ and disappear at $V=690 \mathrm{mV}$. With addition of $C_{\mathrm{d}} *=2 \mathrm{~F}$, the oscillations cease, and only steady states are observed.

As it has been pointed out earlier, the steady states of the system do not change upon varying the time scale parameter, in this case, by applying a finite pseudo-capacitance $C_{\mathrm{d}}{ }^{*}$. Thus, the experimentally determined steady states are, in fact, the unstable states of the originally oscillating system. These experiments indicate that the differential controller can also be applied to construct experimental bifurcations diagrams showing the positions of both stable and unstable steady states. We have carried out experiments in a parameter region where chaotic
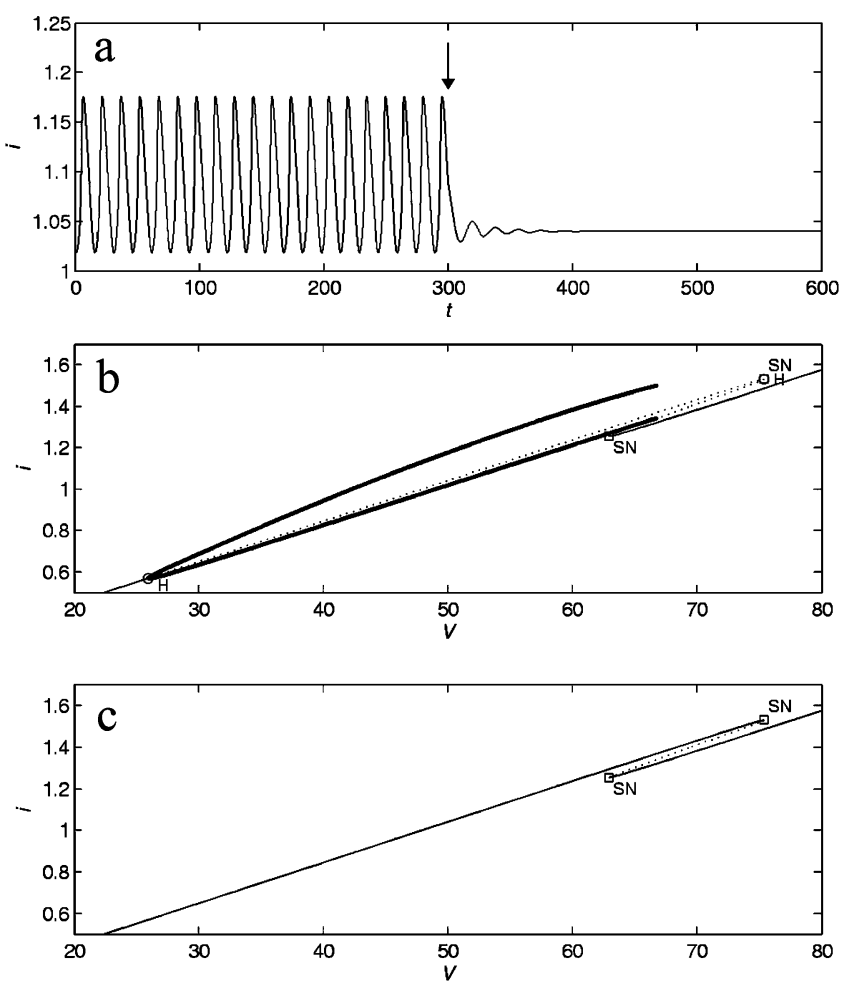

Figure 3. Simulations: Effect of changing the time scale associated with the electrode potential on current oscillations in Ni electrodissolution (HN-NDR system). (a) Suppressing oscillations at $V=50$. At $t$ $=300$ (shown by an arrow) the value of $C_{\mathrm{d}}$ is changed from 1 to 2.5 . (b) Bifurcation diagram as a function of $V$ at $C_{\mathrm{d}}=1$. Annotations are the same as in Figure 1. SN: saddle-node bifurcation. (c) Bifurcation diagram as a function of $V$ at $C_{\mathrm{d}}=2.5$.

oscillations occur. ${ }^{20}$ The chaotic attractor could also be destroyed by the control algorithm, and a stationary state is observed.

3.2. The Effect Pseudo-Capacitance on an HN-NDR Oscillator: Nickel Electrodissolution in Sulfuric Acid. For the electrodissolution of nickel in sulfuric acid the electrode potential was proposed to play the role of a fast activator (similar to $\mathrm{Cu}$ dissolution). However, in this system, the negative differential resistance character of the polarization curve is hindered by a slow process, and thus the negative resistance can be observed only on the time scale of the oscillations. ${ }^{8,9}$ Such systems are known to produce a complicated bifurcation diagram with the possibility of saddle-loop bifurcations. We test the effect of changing the time scale of oscillations in a parameter region where saddle-loop bifurcations are known to occur.

Numerical Simulations. Oscillatory solutions of the model of $\mathrm{Ni}$ electrodissolution can also be suppressed by increasing the double-layer capacitance (Figure 3a). Bifurcation diagrams with respect to circuit potential at low and high values of $C_{\mathrm{d}}$ are shown in Figure 3, parts b and c, respectively. With small $C_{\mathrm{d}}$, the oscillations develop through a supercritical Hopf bifurcation; however, they disappear with a saddle-loop bifurcation associated with the formation of a homoclinic orbit. For large values of $C_{\mathrm{d}}$, the unstable steady state originating from the Hopf bifurcation can be stabilized not only in the region of inherent oscillations but also well over that until it disappears through a saddle-node bifurcation (compare parts $\mathrm{b}$ and $\mathrm{c}$ of Figure 3). Note that the stability of the saddle point does not change with changing the double-layer capacitance; thus, the differential controller is not capable of stabilizing saddle points. Parts $\mathrm{a}$ and $\mathrm{b}$ of Figure 4 show, in a manner similar to that found in the former case of $\mathrm{Cu}$ electrodissolution, that upon 

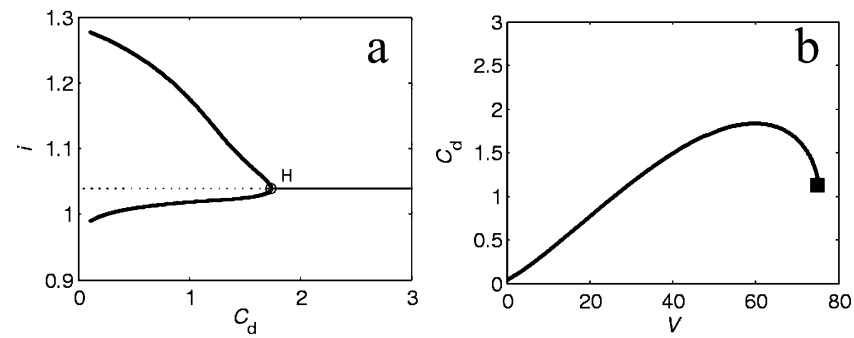

Figure 4. Simulations: Effect of changing the time scale associated with the electrode potential on current oscillations in Ni electrodissolution (HN-NDR system). (a) One parameter bifurcation diagram as a function of $C_{\mathrm{d}}$ at $V=50$. Annotations are the same as in Figure 1. (b) Two parameter bifurcation diagram showing the locus of Hopf bifurcations; solid square: Takens-Bogdanov bifurcation (collision of Hopf and saddle-node bifurcation points).

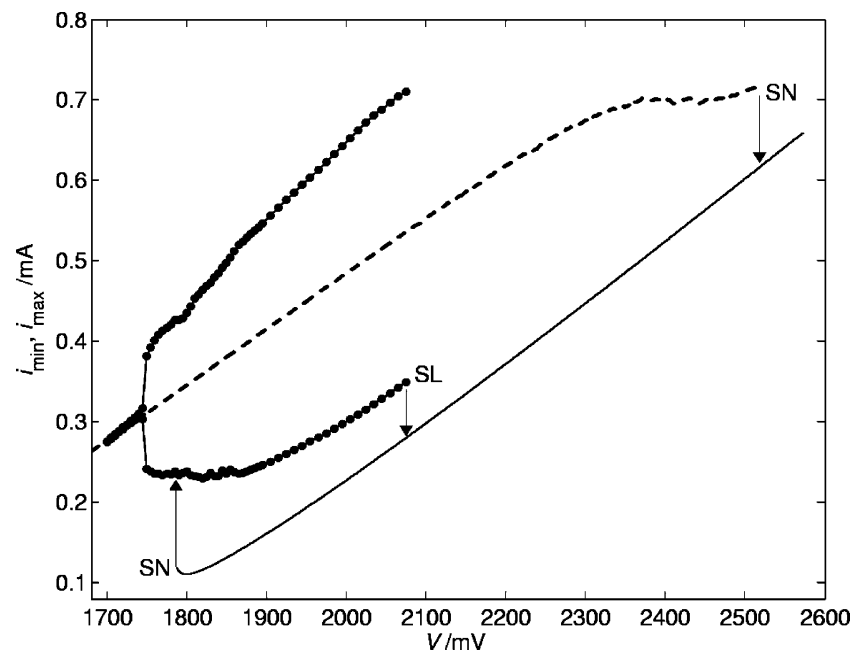

Figure 5. Experiments: Effect of changing the time scale associated with the electrode potential on current oscillations in Ni electrodissolution (HN-NDR system). (a) Polarization curve at $R_{\Omega}=1200 \Omega$ without and with added pseudo-capacitance (solid and dashed line, respectively) $C_{\mathrm{d}} *=0.694 \mathrm{~F}$ (scan rate: $0.8 \mathrm{mV} / \mathrm{s}$ ). For oscillations only the current minima and maxima are shown; annotations are the same as in previous figures; SL-saddle-loop bifurcation.

increasing the time scale variable of the double-layer potential, the current oscillations are suppressed through Hopf bifurcation. There also exists a maximal value of $C_{\mathrm{d}}$ above which no oscillations are possible independently of the applied circuit potential. The locus of the Hopf bifurcation points in the two parameter bifurcation diagram (Figure 4b) is different from that found in the model of $\mathrm{Cu}$ electrodissolution. Here, the locus ends abruptly because of a Takens-Bogdanov bifurcation (collision of Hopf and saddle-node bifurcation points) takes place.

Experiments. Similar to the experiments of copper electrodissolution, the current oscillations during Ni dissolution can be suppressed by addition of pseudo-capacitance on the order of $0.1-1 \mathrm{~F}$. Figure 5 shows the results of scan experiments without and with added pseudo-capacitance in which the circuit potential was slowly increased. Without added capacitance, oscillations appear via a supercritical Hopf and cease through a saddle-loop bifurcation. After the saddle-loop bifurcation a relatively low-current stable state is found only; this state disappears at lower potentials with a saddle-node bifurcation. When the potential scan is performed at $C_{\mathrm{d}} *=0.694 \mathrm{~F}$, no oscillations are observed, and a high-current state prevails until very large values of circuit potential. Our efforts to stabilize the saddle point expected to lie between the high- and lowcurrent steady states were unsuccessful. All these experiments
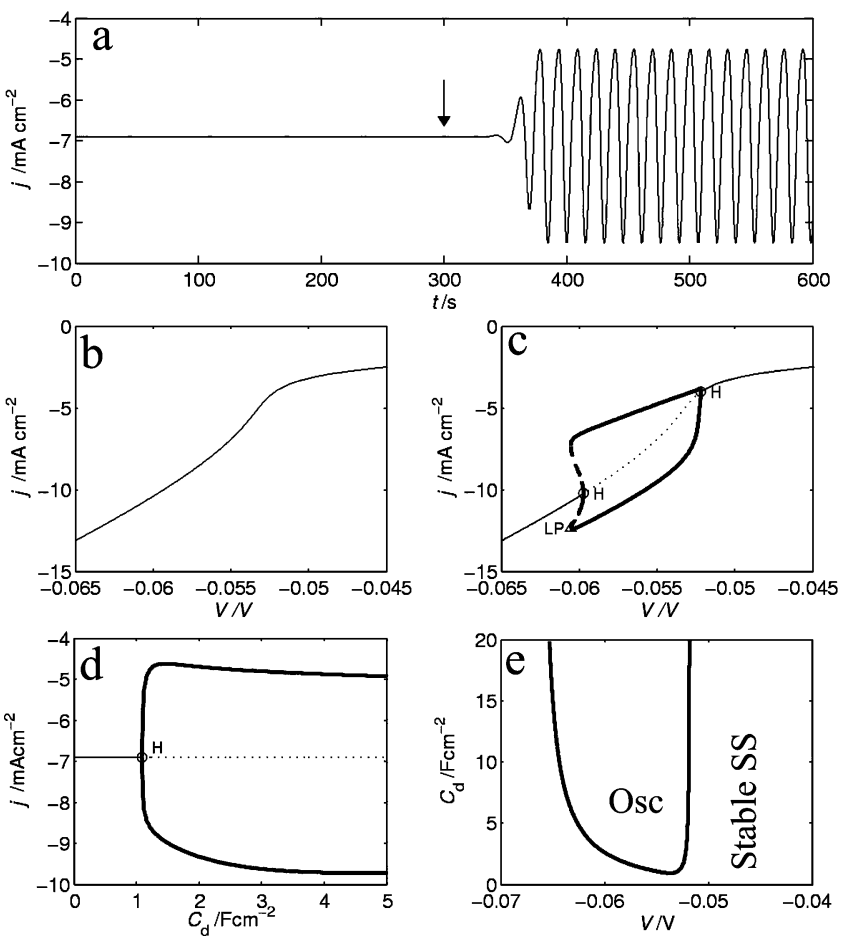

Figure 6. Simulations: Effect of changing the time scale associated with the electrode potential on current oscillations in $\mathrm{Zn}$ electrodeposition (S-NDR system). (a) Inducing oscillations at $V=-55 \mathrm{mV}$ by increasing the value of $C_{\mathrm{d}}$ from $2.5 \mu \mathrm{F} \mathrm{cm}^{-2}$ to $2.5 \mathrm{~F} \mathrm{~cm}^{-2}$ at $t=300$ $\mathrm{s}$ (shown by an arrow). (b) Bifurcation diagram at $C_{\mathrm{d}}=2.5 \mu \mathrm{F} \mathrm{cm} \mathrm{cm}^{-2}$ as a function of $V$. (c) Bifurcation diagram as a function of $V$ at $C_{\mathrm{d}}=$ $2.5 \mathrm{~F} \mathrm{~cm}^{-2}$. LP: saddle-node bifurcation of a periodic orbit. (d) Bifurcation diagram at $V=-55 \mathrm{mV}$ as a function of $C_{\mathrm{d}}$. (e) Two parameter bifurcation diagram showing the locus of Hopf bifurcations.

are in good agreement with the predictions of numerical simulations that in the $\mathrm{Ni}$ system current oscillations can be suppressed with the differential controller and that the saddle points cannot be stabilized.

3.3 The Effect of Pseudo-Capacitance on an S-NDR Oscillator: Zinc Electrodeposition. In S-NDR oscillatory systems the electrode potential plays the role of a slow stabilizer. ${ }^{7,9}$ There are a few examples where the electrode potential acts as a deactivator, most notably $\mathrm{CO}$ electrooxidation, ${ }^{25}$ reduction of periodate ion on gold in the presence of camphor, ${ }^{26}$ and $\mathrm{Zn}$ electrodeposition. ${ }^{14,15}$ However, oscillations are difficult to find because the electrode potential is a fast variable as a result of the small value of double-layer capacitance $\left(\mu \mathrm{F} / \mathrm{cm}^{2}\right)$. We shall apply the differential control algorithm to prove the presence of feedback loops in the mechanism of $\mathrm{Zn}$ electrodeposition and explore the characteristic features of the oscillations.

Numerical Simulations. The model of $\mathrm{Zn}$ electrodepostion exhibits no oscillations at low $C_{\mathrm{d}}$ values. However, current oscillations can be induced by increasing $C_{\mathrm{d}}$ as shown in Figure 6a. By comparing the calculated bifurcation diagrams as a function of the circuit potential at low and high $C_{\mathrm{d}}$ values (see Figure 6, parts $b$ and c), one can see that upon decreasing the cathodic overpotential the oscillations develop through a supercritical Hopf bifurcation and cease via a saddle-node bifurcation of the periodic orbits (following a subcritical Hopf bifurcation). By changing $C_{\mathrm{d}}$ at a fixed circuit potential (Figure $6 d)$ the oscillations appear through a Hopf bifurcation at a relatively large value of the double-layer capacitance $\left(1 \mathrm{~F} / \mathrm{cm}^{2}\right)$. In contrast to that observed in the case of $\mathrm{Cu}$ and $\mathrm{Ni}$ electrodissolution, now a minimal value of $C_{\mathrm{d}}$ is found (Figure 

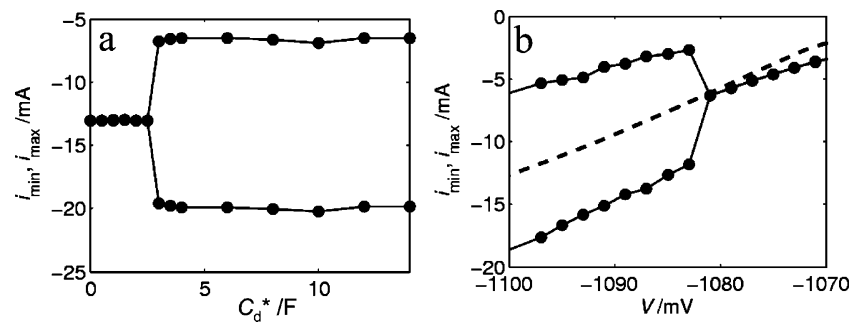

Figure 7. Experiments: Effect of changing the time scale associated with the electrode potential on current oscillations in $\mathrm{Zn}$ electrodeposition (S-NDR system). (a) One parameter bifurcation diagram at $V_{\mathrm{o}}$ $=-1100 \mathrm{mV}$ and $R_{\Omega}=9.7 \Omega$ showing the minima and maxima of current oscillations as a function of the pseudo-capacitance $C_{\mathrm{d}}{ }^{*}$. (b) One parameter bifurcation diagrams at $R_{\Omega}=9.7 \Omega$ with respect to the circuit potential $V$ : dashed line, $C_{\mathrm{d}}{ }^{*}=0 \mathrm{~F}$; solid line, $C_{\mathrm{d}}{ }^{*}=20.0 \mathrm{~F}$.

6e). Below this value, no oscillations are possible. It is also seen from the figure that larger pseudo-capacitance results in wider potential region for oscillations.

Experiments. Current oscillations are induced during Zn electrodissolution by using the differential control algorithm such that pseudo-capacitance is generated in the order of few Farads. Figure $7 \mathrm{a}$ shows that oscillations of nearly constant amplitude occur above $C_{\mathrm{d}}{ }^{*}=2.5 \mathrm{~F}$; both the magnitude of $C_{\mathrm{d}} *$ and the qualitative features of the oscillations are similar to those predicted by the numerical simulations (see Figure $6 \mathrm{~d}$ for comparison). Figure $7 \mathrm{~b}$ shows the maxima and minima of oscillations with increasing overpotential at a fairly large value of $C_{\mathrm{d}}$. (The dashed line shows the steady-state current measured with no pseudo-capacitance.) At about $-1082 \mathrm{mV}$, current oscillations appear that are stable until the potential values are decreased to as low as $-1100 \mathrm{mV}$ (experiments below -1100 $\mathrm{mV}$ were less reproducible because of some nonstationary processes resulting in large increase of cathodic current). The experimental observations are in excellent agreement with the results of numerical simulations predicting that the electrode potential plays the role of a slow stabilizer in the oscillatory feedback loop provided that its variation is slowed by adding pseudo-capacitance.

\section{Discussions}

Investigation of oscillatory chemical reactions is a demanding task that typically requires a combination of devoted experimental and numerical studies. First, one has to collect as much information on the dynamical behavior of the system as possible. Then, the reaction mechanism (and kinetic equations) must be constructed with "reasonable" details that reproduce the observations. Our work contributes to the first step. Traditionally, dynamical information is limited to the observation of stable states and their qualitative change as some parameters are varied. Techniques have been develop to extract various dynamical information: quenching can be used to determine eigenvalues and eigenvectors; ${ }^{27}$ procedures are being developed to reconstruct the Jacobian matrices from pulse perturbations and delayed feedbacks ${ }^{4,28}$ and to identify bifurcations from direct experiments. ${ }^{29,30}$ Modern control techniques can also be applied to stabilize unstable periodic orbits ${ }^{20,31}$ and steady states. ${ }^{32}$

In this study, we applied a differential controller to oscillatory chemical reactions in order to gain direct dynamical information. The major role of the controller is to change the time scale associated with a variable for which it has been applied. Obviously, if the controlled variable is dynamically nonessential, the controller does not affect the oscillatory dynamics. Thus, the differential controller provides direct information on whether a certain chemical species (or a variable) is essential or not. Since most oscillations require the appropriate timing of the slow stabilizing and the fast destabilizing feedback loops, changing the time scale of variation of a single species can have strong effect on the dynamics. On one hand, slowing-down fast destabilizing species or speeding-up slow stabilizing species can destroy oscillations. We demonstrated the suppression of current oscillations during $\mathrm{Cu}$ and $\mathrm{Ni}$ electrodissolution by slowingdown the variation of electrode potential. Since the mechanism of the destruction of the oscillations is general, it is independent of the type of bifurcation through which the oscillations developed; we have demonstrated the cessation of oscillations that were created by Hopf or saddle-loop bifurcations and in some cases by saddle-node bifurcations of periodic orbits. On the other hand, we also demonstrated (with studying $\mathrm{Zn}$ electrodeposition) that oscillations can be induced by changing the time scale of the variation of a variable that is originally too fast for oscillations to arise. Thus, the proposed method could find application in designing oscillators; there seems to be a renewed interest in designing oscillatory chemical systems $\mathrm{s}^{33}$ for potential applications in drug delivery ${ }^{34,35}$ and chemomechanical gel systems. ${ }^{36,37}$

We have also applied the proposed control method for the classification of oscillating electrochemical systems. There exist a surprisingly large number of oscillatory electrochemical reactions mainly because of the important role of the electrode potential. ${ }^{8,9}$ An operational approach to the classification was proposed earlier based on the role of electrode potential. ${ }^{7}$ The classification requires the investigation of the system's dynamics as a function of added external resistance. Here, we suggest a complementary approach based on a differential controller that can be used as a versatile tool for the task of identification. The recursive feedback controller can be applied to add (positive or negative) pseudo-capacitance to the inherent double-layer capacitance of the system. We have demonstrated that in systems of which the electrode potential plays the role of a fast activator species (N-NDR and HN-NDR type) the added pseudocapacitance destroys the oscillations through Hopf bifurcation. We also demonstrated that in an electrochemical system in which the electrode potential plays the role of slow inhibitor (S-NDR) current oscillations occur at large capacitance values only. The qualitatively different behavior is indicative of basic difference in the underlying kinetics. Derivative controller was previously used to study the dynamics of $\mathrm{H}_{2}$ electrooxidation; ${ }^{38}$ however, in that study no recursive algorithm was used for the feedback, and thus the information related to the reaction mechanism is limited.

Our numerical simulations are in excellent agreement with the experimental observations about the effect of changing the double-layer capacitance. Since the double-layer capacitance is a vital parameter in these models, the agreement between calculations and experiments is a strong indication that the theory of electrochemical oscillators ${ }^{8,9}$ - considering the effect of electrode potential-is on strong fundamental basis.

The addition of pseudo-capacitance was carried out with a feedback controller; this provides a wide range of capacitance values limited only by the stability of the algorithm. (Note that even negative capacitance can be implemented.) Pseudocapacitance can also be generated chemically; it can arise at an electrode when the extent of the faradaically admitted charge depends linearly (or approximately linearly) on the applied voltage: this capacitance is faradaic in origin and not electrostatic. ${ }^{39}$ Two known types of materials that exhibit this behavior are conducting polymers and $\mathrm{RuO}_{2}{ }^{40}$ The effect of changing 
the capacitance of an electrode by polyaniline film was described in galvanostatic oscillations during formic acid oxidation. ${ }^{41}$ The application of these materials to design electrochemical oscillators is a promising option but hardship could arise due to the required large changes in the capacitance; our method allows effects on the order of few $\mathrm{F} / \mathrm{cm}^{2}$ while materials exhibit pseudocapacitances on the order of few $\mathrm{mF} / \mathrm{cm}^{2}$ only. ${ }^{40}$

Although the application of changing time scales with differential controller was demonstrated with electrochemical systems, it is certainly not limited to this field of interest. Feedback controllers can be designed, for example, for continuously fed stirred reactors where the inlet concentrations of chemicals could be changed proportional to the concurrently determined time derivative of concentrations. Such studies would give direct information on the role of essential species and thus provide valuable information about the oscillatory dynamics. The approach could also be applied to biological systems where rhythms, generated typically by large reaction networks of limited kinetic information, are widespread. ${ }^{42}$

Acknowledgment. We acknowledge OTKA (T038071) for financial support.

\section{References and Notes}

(1) Tyson, J. J. J. Chem. Phys. 1975, 62, 1010.

(2) Clarke, B. L. Adv. Chem. Phys. 1980, 43, 1.

(3) Eiswirth, M.; Freund, A.; Ross, J. Adv. Chem. Phys. 1991, 80, 127.

(4) Chevalier, T.; Schreiber, I.; Ross, J. J. Phys. Chem. 1993, 97, 6776.

(5) Strasser, P.; Eiswirth, M.; Ertl, G. J. Chem. Phys. 1997, 107, 991.

(6) Epstein, I. R.; Pojman, J. A. An Introduction to Nonlinear Chemical Dynamics: Oscillations, Waves, Patterns, and Chaos; Oxford University Press: Oxford, U.K., 1998.

(7) Strasser, P.; Eiswirth, M.; Koper, M. T. M. J. Electroanal. Chem. 1999, 478,50 .

(8) Koper, M. T. M. Adv. Chem. Phys. 1996, 92, 161.

(9) Krischer, K. Principles of temporal and spatial pattern formation in electrochemical systems. In Modern Aspects of Electrochemistry; Conway, B. E., Bockris, O. M., White, R. E., Eds.; Kluwer Academic/Plenum Press: New York, 1999; Vol. 32; p 1.

(10) Hudson, J. L.; Tsotsis, T. T. Chem. Eng. Sci. 1994, 49, 1493.

(11) Kiss, I. Z.; Zhai, Y.; Hudson, J. L. Phys. Rev. Lett. 2002, 88, 238301.

(12) Albahadily, F. N.; Schell, M. J. Chem. Phys. 1988, 88, 4312.

(13) Lev, O.; Wolffberg, A.; Sheintuch, M.; Pismen, L. M. Chem. Eng. Sci. 1988, 43, 1339.

(14) Epelboin, I.; Ksouri, M.; Lejay, E.; Wiart, R. Electrochim. Acta 1975, 20, 603

(15) Epelboin, I.; Ksouri, M.; Wiart, R. J. Electrochem. Soc. 1975, 122, 1206.
(16) Bressan, J.; Wiart, R. J. Appl. Electrochem. 1979, 9, 43.

(17) Stephanopoulos, G. Chemical Process Control: An Introducation to Theory and Practice; Prentice Hall: Englewood Cliffs, NJ, 1984.

(18) Parmananda, P.; Eiswirth, M. J. Phys. Chem. 1996, 100, 16568.

(19) Koper, M. T. M.; Gaspard, P. J. Chem. Phys. 1992, 96, 7797.

(20) Kiss, I. Z.; Gáspár, V.; Nyikos, L.; Parmananda, P. J. Phys. Chem. A 1997, 101, 8668 .

(21) Kiss, I. Z.; Gáspár, V.; Nyikos, L. J. Phys. Chem. A 1998, 102, 909.

(22) Haim, D.; Lev, O.; Pismen, L. M.; Sheintuch, M. J. Phys. Chem. 1992, 96, 2676.

(23) Lee, M. G.; Jorné, J. J. Electrochem. Soc. 1992, 139, 2841.

(24) Ermentrout, B. Simulating, Analyzing, and Animating Dynamical Systems: A Guide to XPPAUT for Researchers and Students; SIAM: Philadelphia, PA, 2002.

(25) Koper, M. T. M.; Schmidt, T. J.; Markovic, N. M.; Ross, P. N. J. Phys. Chem. B 2001, 105, 8381 .

(26) Li, Y. J.; Oslonovitch, J.; Mazouz, N.; Plenge, F.; Krischer, K.; Ertl, G. Science 2001, 291, 2395.

(27) Hynne, F.; Sorensen, P. G. Phys. Rev. E 1993, 48, 4106.

(28) Mihaliuk, E.; Skodt, H.; Hynne, F.; Sorensen, P. G.; Showalter, K. J. Phys. Chem. A 1999, 103, 8246.

(29) Schreiber, I.; Ross, J. J. Phys. Chem. A 2003, 107, 9846.

(30) Rico-Martinez, R.; Krischer, K.; Flatgen, G.; Anderson, J. S.; Kevrekidis, I. G. Physica D 2003, 176, 1.

(31) Petrov, V.; Gáspár, V.; Masere, J.; Showalter, K. Nature (London) 1993, 361, 240.

(32) Pyragas, K.; Pyragas, V.; Kiss, I. Z.; Hudson, J. L. Phys. Rev. Lett. 2002, 89, 244103.

(33) Kurin-Csörgei, K.; Epstein, I. R.; Orbán, M. Nature (London) 2005 , $433,139$.

(34) Misra, G. P.; Siegel, R. A. J. Pharm. Sci. 2002, 91, 2003.

(35) Misra, G. P.; Siegel, R. A. J. Controlled Release 2002, 79, 293.

(36) Crook, C. J.; Cadby, A.; Smith, A.; Jones, R. A. L.; Ryan, A. J. Measurement of force produced by a $\mathrm{pH}$-responsive hydrogel in a $\mathrm{pH}$ oscillator. In Nonlinear Dynamics in Polymeric Systems; Pojman, J. A., Tran-Cong-Miyata, Q., Eds.; ACS Symposium Series 869; American Chemical Society: Washington, DC, 2004; p 71.

(37) Gauffre, F.; Labrot, V.; Boissonade, J.; De Kepper, P. Spontaneous deformations in polymer gels driven by chemomechanical instabilities. In Nonlinear Dynamics in Polymeric Systems; Pojman, J. A., Tran-CongMiyata, Q., Eds.; ACS Symposium Series 869; American Chemical Society: Washington, DC, 2004; p 80.

(38) Strasser, P.; Lübke, M.; Parmananda, P.; Eiswirth, M.; Ertl, G. $J$ Phys. Chem. B 1998, 102, 3227.

(39) Bockris, J. O. M.; Reddy, A. K. N. Modern Electrochemistry; Plenum Press: New York, 1973; Vol. 2.

(40) Conway, B. E. Electrochemical Supercapacitors: Scientific Fundamental and Technological Applications; Kluwer Academic/Plenum Publishing: New York, 1999.

(41) Inzelt, G.; Kertész, V. Electrochim. Acta 1997, 42, 229.

(42) Winfree, A. T. The geometry of biological time; Springer-Verlag: New York, 1980. 